\title{
Conceptual coordination bridges information processing and neurophysiology
}

\author{
William J. Clancey \\ Computational Sciences Division, M/S 269-3 \\ NASA/Ames Research Center \\ Moffett Field, CA 94035 \\ bclancey@mail.arc.nasa.gov \\ http://ic.arc.nasa.gov/ic/clancey.html
}

\begin{abstract}
Information processing theories of memory and skills can be reformulated in terms of how categories are physically and temporally related, a process called conceptual coordination. Dreaming can then be understood as a story understanding process in which two mechanisms found in everyday comprehension are missing: conceiving sequences (chunking categories in time as a higher-order categorization) and coordinating across modalities (e.g., relating the sound of a word and the image of its meaning). On this basis, we can readily identify isomorphisms between dream phenomenology and neurophysiology, and explain the function of dreaming as facilitating future coordination of sequential, cross-modal categorization (i.e., REM sleep lowers activation thresholds, "unlearning").
\end{abstract}

\section{COMMENTARY ON BBS SPECIAL ISSUE ON SLEEP AND DREAMING}

Now is a good time to bridge the different disciplines of the cognitive and neurosciences on the issue of dreams, with far-reaching implications for future theorizing across disciplines. But relating information processing theories to dream phenomenology and neurophysiology requires understanding the inherent, temporal basis of memory. In turn, a theory of consciousness can be developed that foregrounds how categories are constructed sequentially, cross-modally, and hierarchically in time (Clancey 1999), supported by Hobson, Pace-Schott, and Stickgold's (HP\&S) analysis of REM neurophyisology. From a connectionist perspective, the disinhibition of cross-modal activations suggests "reverse learning" (Crick \& Mitchison 1983), by which the neural network is settling down to allow new associations to form or to lower the threshold required to coordinate experience sequentially. Thus procedural memory cannot be coordinated in REM sleep (ruling out any complex rehearsal of survival skills, contra Revonsuo). Instead, the function is to facilitate future learning in the awake state (contra Vertes and Eastman). Attempts to relate dreams to REM and NREM neurophysiology (Solms and Nielsen) can be improved by characterizing how categories are related in sleep experiences. 
What aspects of memory are missing? Dream phenomenology provides striking clues about the neurophysiology of REM sleep as well as aspects of memory and categorization that are essential for everyday consciousness. Perhaps of most importance are scene shifts and multi-modal discoordinations, which are taken for granted by the dreamer (HP\&S, sec II.C4). Freud (1900) characterized this phenomenology in terms of a rebus puzzle. For example, in a dream I see a stick in the ground and say to myself "I have a lot at stake" and next am eating a steak. The meaning of the dream to me is revealed by my description, not the literal images or incidents (thus dream structure-the mix of images, sounds, and ideas - is organized by a verbal conceptualization of important concerns in my life, what I have "at stake").

However, to explain dream phenomenology in terms of neurophysiology, we need to characterize and relate both dream content and neurophysiology through an intermediate description of cognitive structure and temporal relationships (Clancey 1999). Finding an isomorphism between dream content and neurophysiology requires reformulating memory and learning in terms of categorization operating upon itself, eschewing notions of a random-access storehouse of beliefs and procedures (Clancey 1997).

Similarly, HP\&S's AIM model can be reformulated in terms of a categorization coordinating mechanism. The notion of "information" is characterized in neuropsychological terms as categorization (Edelman 1987); and "processing" is characterized as kinds of constructive operations by which multi-modal categories are physically and temporally related. Thus, I propose a three-layer analysis by which cognitive aspects of dreams and neurophysiology can be related:

\title{
I Dream Content
} (phenomenology)

\section{Conceptual Coordination Analysis (structural and temporal relations of categorizing)}

\author{
III Neurophysiology Analysis \\ (neural activation between brain areas)
}

HP\&S (sec II.C4) are right that a deficiency in memory goes a long way toward explaining orientational instability, loss of self-reflective awareness, and failure of directed thought and attention. However, the explanation is incomplete until we say more about what aspect of memory is relevant to these aspects of higher-order consciousness. This is the purpose of Level II in my analysis. What specific aspects of memory are missing?

Reformulating cognitive experience in terms of conceptual coordination, we find that higher-order consciousness (e.g., involving directed thought) requires three higher-order categorizing relations that are missing from REM sleep: 1) sequential correlation in multi-modal perceptual categorizing (e.g., relating sound 
and image), 2) holding a category active so it may be compared, counted, contrasted, etc., 3) categorizing a sequence of experience as a conceptual unit (chunking working memory). (See HP\&S Figure 7, areas 4, 7, 8, 10.)

HP\&S's work nicely shows that the missing aspects of higher-order consciousness are due to aminergic demodulation. Or in conceptual coordination terms, the neurological mechanisms by which associations in different modalities are correlated or made consistent (sounds, images, and meanings correspond), by which categories are deliberately related, and by which episodes are held active (so they may be objectified, named, and related) are not operating during REM sleep because of failures in aminergic neuromodulation.

In short, by viewing cognitive processes (information processing) in terms of how categories are formed and related (sequentially, hierarchically) the phenomenological structure of dreams can be explained. And by viewing these cognitive processes in terms of neurophysiology, their absence in REM sleep can be explained. This tripartite approach is essential because otherwise the phenomenology of dreams can only be loosely characterized in terms of "thought" or "episodes," and the role of the neurophysiological processes in everyday cognition will not be sufficiently articulated. As HP\&S (sec IV.A3) imply, psychology has heretofore failed to document the differences between waking and dreaming, just as it failed to document different kinds of consciousness among species, let alone between people and machines. This failure is rooted in a storage view of memory (with properties like copying and simultaneous multiple use of categories) and a verbally dominated view of thought (e.g., the assumption that visual thought and analogical reasoning only occurs by representing images as named objects and relationships, cf. Larkin \& Simon 1987).

Relying on the information processing perspective of cognitive theory (such as a storage and retrieval view of memory), HP\&S's analysis is necessarily limited to talk about information instead of coordinated categorization in time. We can now reformulate AIM in conceptual coordination terms:

Activation (Information Processing Capacity) $\rightarrow$ perceptual categorization, scenes (coupled or synchronous categorizations), sequencing categorizations (episodes), holding a category active, holding a sequence of recent categorizations active (working memory), substitution within a sequence (e.g., saying "chocaholic" by analogy to "alcoholic"), categorizing a sequence (chunking, proceduralization), hierarchical activation of categories (bottom-up and/or topdown).

Input Source $\rightarrow$ perceptual categorization driven by: (external) sensory system, emotional correlation (e.g., dramatic theme such as "End of the World fear"), and/or conceptual (higher-order) categorization (e.g., verbal meanings influence imagery). 
Modulation (Mode of IP) $\rightarrow$ how categories are conceptually coordinated, i.e., how activation is modulated by other (higher-order) categorizations that are already active: correlating categories across modalities (especially sound, image, and meaning), counting, seeing-as, narrative, logical categorizing (e.g., implication, contradiction, identity), hierarchical goal-directed problem solving.

The changes in AIM during REM sleep involve an inability to hold a category or sequence of categorizations active (Activation), a mostly internally driven perceptual categorization (Input Source), and inabilities to conceptually coordinate across sensory systems and to categorize sequences (Modulation).

In summary, without the persistence enabled by sequential (and hence) temporal categorizing of the aminergic neurons, there is neither primary coordination sequencing required to follow and formulate procedural relations nor, consequently, secondary categorization (and awareness) of coordination that is occurring. Aminergic neurons are not categorizing sensorimotor activity over time (matrixing with cortical neurons is missing). With the shutdown of REM-off neurons, the reticular system is disinhibited, contributing to the fantastic crossmodal activations of dreams, in which language, sounds, and images are freely associated. Attentional coordination is lost across systems, facilitated by the lack of feedback from sensorimotor interactions in the world. At the same time, the inability to hold non-sequential or non-synchronous categories active and relate them in time (which occurs in the conceptual coordination of higher-order consciousness) enables wild scene shifts.

How is activation of specific brain areas relevant? As we explicate how dreams are generated (what brain areas and paths are engaged), the conceptual coordination analysis can be mapped in more detail onto specific mechanisms involved in different aspects of categorization.

Insofar as dreaming occurs outside of REM sleep, as Solms argues, its story structure may be different from REM dreams. For instance, lucid dreams may combine disorientation and a capability to observe and comment on experience; whether these experiences are simultaneous or sequential is unclear. Building on Solms' (sec 8) analysis, considering the kind of categorizing occurring in the person's experience may provide a clue about which areas are engaged and how they are relating to each other. For example, how are inabilities to hold a category active and to categorize sequences related to the deactivation of dorsolateral prefrontal cortex?

How does conceptual coordination differ during REM and NREM sleep? Nielsen's effort to characterize the mentation in different forms of sleep may be improved by characterizing the organization of cognitive activity in terms of how categories are related. I suggest the order: perceptual categorization, scenes (simultaneous relation of multiple perceptual categories as in seeing a pen and a knee), sequencing (one scent/event follows another), correlation within a 
sequence (e.g., a sound is followed by a causally corresponding image), holding a categorization active (e.g., comparing ideas), and categorization of a sequence (consciousness of "what I'm doing now").

Thus, Nielsen's Figure 1 might be improved by distinguishing the "cognitive processes" (item 4) that are higher-order categorizations missing in dreams (e.g., consolidation, rehearsal, plus forms of discrimination and selective attention) from the simpler relations found in dreams (e.g., perceptual memory activation, orienting/surprise). Aspects of conceptual coordination in sleep mentation can then be reordered (my Figure 1) according to basic categorization (including Nielsen's "preconscious pecursors"), dreaming (scenes and narrative conceptualization), apex dreaming (protracted conceptualization of dramatic themes), and higher-order consciousness (sequentially coordinated ideas with causal and inferenctial relations, i.e., thinking). Because different kinds of conceptual coordination are occurring, it is too coarse to characterize NREM sleep as "more conceptual and thoughtlike." The question remains how thinking in NREM sleep and awake cognition differ.

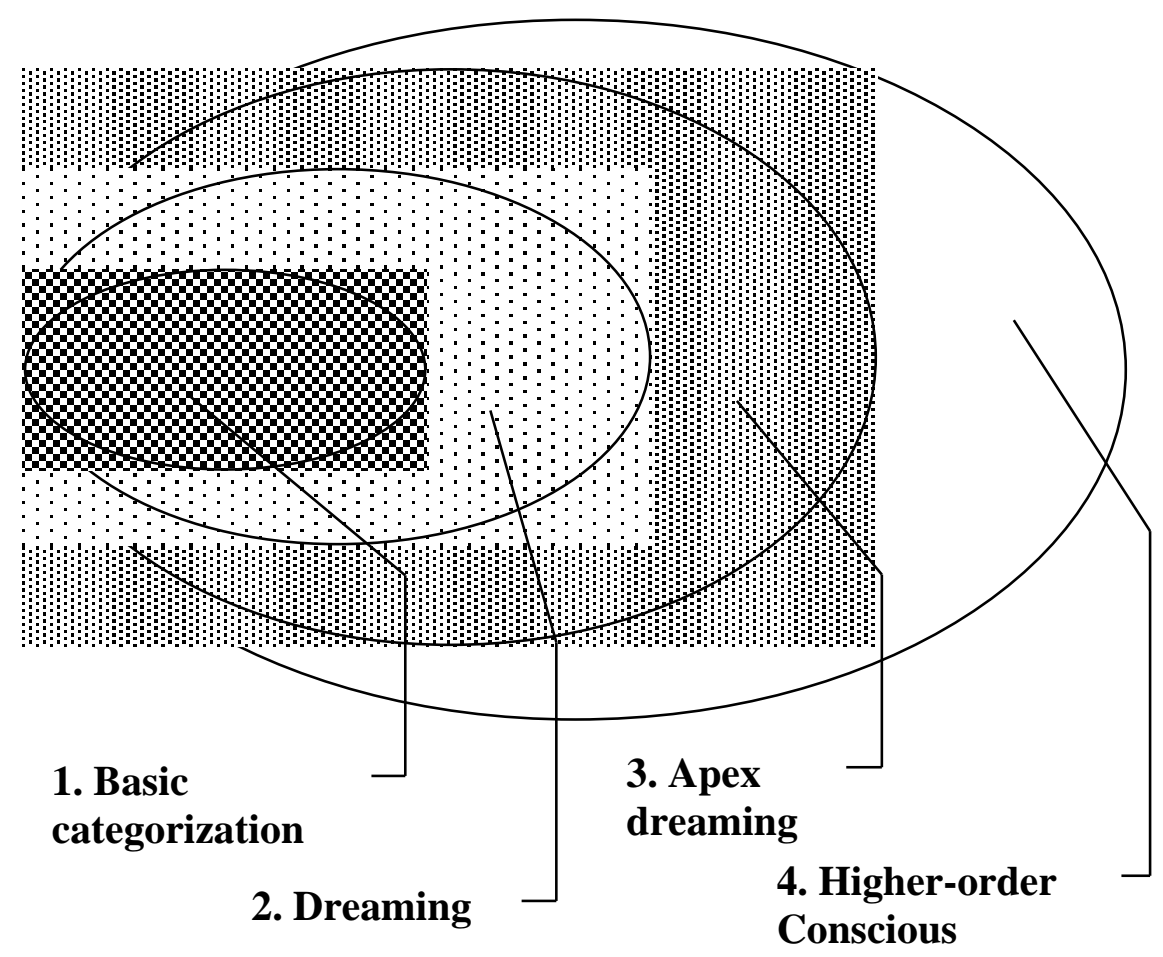

Figure 1. Revision of Nielsen's "levels of specificity" as levels of categorization (increasing conceptual coordination); each oval represents a form of consciousness in which simpler forms of categorization are temporally related in new ways.

What survival skills can be rehearsed without conceputal coordination? On a different level, Revonsuo has provided a broad-ranging, provocative account of the evolutionary function of dreaming. However, we must tighten up the notion of 
what is learned or reinforced and how what is learned relates to awake performance in the everyday environment. Revonsuo's analysis does not adequately distinguish between stimulus/response association and human inference. How could dream experiences, lacking basic aspects of goal-oriented attention, let alone reasoning by analogy and reinterpreting plans, constitute "training episodes" for skilled human performance in threat situations? The structure of dream experience, such as our inability to read text, reveals that conceptual coordination is impaired relative to awake cognitive activity, and hence we can rule out certain evolutionary benefits that require forms of logic, symbolic reference, and analogical reasoning. Although dream content reflects our everyday concerns, the primary function of dreaming, for humans at least, must be neurophysiological.

However, we must proceed carefully. Conceptualization of meaning occurs in dreams without the associated summarizing and encapsulating statements of meaning by which reasoning occurs when awake. The restricted consciousness of dreaming allows formation of new "dream thoughts," but without the elaborated structure of causally coherent narrative and planning that higher-order consciousness allows. The effect of such experience on the awake planning of humans is unclear.

A both-and theory is required: Dream phenomenology is both "the consequence of an active and organized process" and "a passive byproduct of disorganized activation" ( $\sec 3.3$ ). The coherence of dream drama is most definitely not like the sequence coherence of a narrative story or extended episode of experience. Although a dream may have an overarching theme or setting, the co-presence of dream elements (people, objects, and events) and the shifting story line is fundamentally unlike the coherence a person experiences (and indeed insists upon) when awake. Reading, writing, and calculating are absent because a dreaming person is unable to coordinate imagery and verbalization with a calculus. Such skills require procedural coordination (goal-directed, sequential behavior that is hierarchically organized with categorization "bindings" that may be substituted or generalized as behavior occurs; see Clancey 1999). Dream experiences are indeed multi-modal, but they are not sequentially coordinated and therefore cannot be simulations of real experience. Dream experience lacks higher-order consciousness ("insight into our true condition," sec 3.6.1) — precisely what we rely upon to respond flexibly to threats in real-life.

Human cognition is not just a stimulus-response system. Response to threats is not merely a matter of fight or flight. People anticipate (imagine what will happen next), plan (imagine what they might do next), make weapons (organize tools and get ready for some action). The complex behaviors involved in hunting and defending a habitat, especially in a social manner, are indeed skills. But they involve a kind of coordinated representation, reification, organization of materials, and behavior sequencing that are not possible during REM consciousness. What kind of simulations might be useful? Logical thinking! 
Contrast the dream experience "stung by bees" with the skills of recognizing bee nests or areas where they might gather, methods of killing bees, getting honey from a bee hive, and interpreting how bee behaviors relate to climate and seasons. Aside from merely reinforcing a flight response, a dream about bees could at best reinforce a person's interest to learn more about bees or to attend to associated bee phenomena when awake.

One implication of Revonsuo's theory is that dreaming reinforces an unthinking way of responding to threat situations, merely based on reactive, perceptually and emotionally driven behavior. If this was indeed an evolutionary advantage, it was originally conferred on other mammals, not Pleistocene Man. Such learned associations, if any, are not like skilled human knowledge, because they are not procedurally integrated and flexibly controlled.

The presence of realistic imagery is not sufficient, there must be deliberate behavior, namely sustained attention that holds a goal in mind and orients interpretation and action in a coordinated way to accomplish the goal. In a daydream we can imagine a sequence of events and actions, with controlled behavior. But we lack this capability when dreaming.

Examples of "implicit learning" when awake merely show that correlations and sequential relations may be learned without reifying them into named objects and relations that are reasoned about (Clancey 1999). Nevertheless, the person is paying attention and performing with higher-order control. Indeed, dream experience lacks the correlation of incidents that is meaningful when awake, so how could a dream sequence produce a useful expectation of how events will unfold in awake life?

Revonsuo cites evidence that REMD impairs memory for procedural or implicit tasks, which again supports the hypothesis that REM involves a relaxation/unlearning effect that facilitates later learning. So the benefit of REM would be to facilitate future learning in real-life situations, not to rehearse those situations during the dream itself.

At another level, if the average ancestral human were constantly confronted with threatening events ( $\sec 3.8 .1$ ), why would they need to be rehearsed? Everyday experience would surely provide enough practice to develop well-honed, adapted skills. Similarly, Revonsuo views survival skills too narrowly in terms of immediate physical dangers. Aren't "underrepresented peaceful activities," such as working in a forest, as important for survival as appropriate response to threats?

Does REM sleep facilitate future procedural learning? Vertes \& Eastman's (V\&E) thesis is strongly supported by a conceptual coordination perspective that processing and consolidation of experience requires aspects of consciousness that 
are missing during REM sleep. But the conclusion that REM sleep could only serve to maintain CNS activity during sleep is not warranted.

V\&E cite effects of post-REM deprivation on performance of an already practiced task (sec 2.5) that appear consistent with the hypothesis that REM sleep "settles" the activation level of cross-modal coordination, thus lowering the threshold required for stimulation and hence improving performance. This in itself does not show that consolidation is operating. Instead, performance when awake could be enhanced by the clarity of mind that results from a lowered threshold required to coordinate behavior, and hence an ease in reconstructing practiced skills. For example, sitting down to play the piano in the morning, you may find that the passages you labored over the night before are now effortlessly recollected. The practiced behaviors reactivate on an uncluttered path, as irrelevant relations, such as the conceptual context in which the practice sessions occurred, are not salient.

V\&E cite other REMD studies supporting the hypothesis that REM prepares the brain for future multi-modal coordination learning ( $\mathrm{sec} 2.2 .2$ ). Further experiments might accordingly focus on learning involving multi-modal coordination such as sight-reading music, text comprehension involving visualization and calculation, or navigation involving multisensory cues and spatial orientation. (See also studies of REM sleep integrity and duration cited by Nielsen, sec 2.2, which provide related support.)

What does dreaming reveal about consciousness? Perhaps the most exciting result of this analysis is what it reveals about consciousness. First, we are conscious when we dream-a major shift from the idea that sleep is an "unconscious" state. Second, story comprehension-making sense of experience through narrative conceptualization - is more fundamental than logical thought (Donald 1991). Third, the essential coordination abilities of awake human consciousness are to hold a category active as a kind of anchor (e.g., to find a correlate and thus to have a basis of a higher-order relational categorization, such as " $\mathrm{x}$ is bigger than $\mathrm{y}$ ") and to hold a sequence active and categorize it (e.g., to conceive of an episode, a procedure or method). These neuropsychological processes enable formulating goals and means for accomplishing them.

Now we may more fruitfully inquire about cognition in other animals. Do all primates have the conceptual coordination capabilities described here? Is counting possible without being able to hold a category active (e.g., scanning objects and incrementing the total)? Do other animals experience in their awake state the rapid scene shifts of human dreams? Does language confer a more stable way of holding a category active, such that cats may be goal directed, but be easily distracted and fooled because they do not name their intentions and reason about shifts in their attention? Can some personality dysfunctions (Rosenfield 1992) be reformulated in terms of inability to coordinate a protracted sequence of "what I'm doing now" (evidenced by Mr. T's rapid categorical shifts of "who I am" [Sacks 1987])? 
Such questions are possible only because we no longer take for granted the conceptual coordination capabilities (binding, matching, storing, iterating) that procedural programming languages have given cognitive modelers for free. Reformulating memory, attention, and thought in terms of the neuropsychological mechanisms of consciousness is a dramatic breakthrough-perhaps the most important advance since the information processing revolution in psychology fifty years ago.

\section{REFERENCES}

Clancey, W. J. (1997) Situated cognition: On human knowledge and computer representations. Cambridge University Press.

Clancey, W.J. (1999) Conceptual coordination: How the mind orders experience in time. Erlbaum.

Crick, F. \& Mitchison, G. (1983) The function of dream sleep. Nature, 304(14, July), 111-115.

Donald, M. (1991) Origins of the modern mind: Three stages in the evolution of culture and cognition. Harvard University Press.

Edelman, G. M. (1987) Neural Darwinism: The theory of neuronal group selection. Basic Books.

Freud, S. ([1900]1965) The interpretation of dreams. Avon Books.

Larkin, J. H. \& Simon, H. A. (1987) Why a diagram is (sometimes) worth ten thousand words. Cognitive Science, 11(1), 65-100.

Rosenfield, I. (1992) The strange, familiar, and forgotten. Vintage Books.

Sacks, O. (1987) The man who mistook his wife for a hat. Harper \& Row. 